\title{
31. The Observation of Right Ascension and the Determination of Proper Motion of Zodiacal Stars.
}

\author{
By Kônosuke TuzI. \\ Contribation from the Tokyo Astronomical Observatory. \\ (Comm. by Y. HagiharA, M.I.A., March 12, 1945.)
}

The observation of the right ascension of 2790 zodiacal stars has been carried out by the present author with the Repsold transit instrument in the Tôkyô Astronomical Observatory. Their proper motions have been determined and a starcatalog of these stars has been compiled on the basis of this observation. The method of procedure and the brief summary of the result are reported in the present note.

1. The zodiacal stars and the system of reference.

David Gill has published the list of zodiacal stars selected in such manner that the position of any star within the zodiacal zone could be accurately determined from these selected zodiacal stars by the differential method. In our observation 97 stars contained both in the $F K 3$ catalog and in Gill's list of zodiacal stars have been adopted as the clock stars among our 2790 stars, and the places of such stars have been computed from the $F K 3$ catalog. Thus the observed right ascensions of our zodiacal stars are all referred to the $F K 3$ system. Naturally the clock stars are limitted to the zodiacal zone.

2. The observation of right ascension of zodiacal star.

The detail of the instrument, the method of observation and the individual result of each night have been already reported in the Tôkyô Astronomical Bulletin Nos. 273-688. In each night more than ten clock stars, two azimuth stars and zodiacal stars of our program were observed, and the telescope was reversed before or after the observation, in order to determine the collimation by means of the two collimators installed at two meters' distance from the instrument. The level error was read off by a hanging level, several times during each set of the observation.

The apparent places of the clock stars were calculated, and the accuracy of the observation was examined every night by computing the probable error of the clock correction of the time keeper directly used for the observation. The mean value of the probable error of the clock correction by a single transit is \pm 0.020 , 
and the probable error of the bisection of a single transit is \pm 0.017 . The observation was commenced in November 1937 and completed in October 1942. All program stars have been observed more than four times with 12 exceptions, the probable error of the observed right ascension of each star being less than \pm 0.009 with ten exceptions.

The observed right ascensions at the owserved epoch and the equinox have been reduced to the mean equinox 1950.0 by basing Newcomb's precession constants in accordance with the $F K 3$ system. The results have been reported from time to time in the Tôkyô Astronomical Bulletin No. 273-688. $\Lambda \alpha$ of the observed clock star, which is identical with the mean value of the deviation of the clock correction of each clock star, is rather small. The total mean value of $\boldsymbol{\Lambda} \boldsymbol{\alpha}$. for all the clocir stars is $+0^{\mathrm{s}} 0003$ and their hourly mean is less than $\pm 0^{\mathrm{s}} 006$, and no systematic deviation for the right ascensions has been detected. Hence it

Table I.

$$
\begin{aligned}
\Delta \alpha= & (\alpha \text { in any catalog })-(\alpha \text { in the } F K 3 \text { catalog }) \\
= & x+y \sin \alpha+z \cos \alpha+w \sin 2 \alpha+u \cos 2 \alpha \\
& \text { at the epoch and the equinox } 1900.0
\end{aligned}
$$

\begin{tabular}{|c|c|c|c|c|c|}
\hline & $x$ & $y$ & $z$ & $w$ & $u$ \\
\hline Cape & $\begin{array}{l}\mathrm{s} \\
.0628 \pm .0013\end{array}$ & $\begin{array}{l}\mathrm{s} \\
.0165 \pm .0018\end{array}$ & $\begin{array}{cc}\mathrm{s} & \mathrm{s} \\
-.0051 \pm .0019\end{array}$ & $\begin{array}{l}\mathrm{s} \\
.0939 \pm .0018\end{array}$ & $\begin{array}{l}\mathrm{s} \\
.0100 \pm .0018\end{array}$ \\
\hline Heidelberg & $.0644 \pm .0013$ & $.0161 \pm .0018$ & $-.0039 \pm .0019$ & $.0862 \pm .0019$ & $.0003 \pm .0018$ \\
\hline Washington & $.0419 \pm .0011$ & $-.0103 \pm .0015$ & $.0010 \pm .0015$ & $.0899 \pm .0015$ & $-.0006 \pm .0015$ \\
\hline Lick & $.0605 \pm .0024$ & $.0387 \pm .0033$ & $-.0065 \pm .0035$ & $.0939 \pm .0035$ & $.0009 \pm .0034$ \\
\hline Königsberg & $.0619 \pm .0013$ & $.0043 \pm .0018$ & $-.0098 \pm .0018$ & $.0895 \pm .0018$ & $-.0016 \pm .0018$ \\
\hline Edingburgh & $.0575 \pm .0013$ & $.0055 \pm .0019$ & $-.0090 \pm .0019$ & $.0916 \pm .0019$ & $-.0039 \pm .0019$ \\
\hline ZOD 1900 & $-.0001 \pm .0010$ & $-.0025 \pm, 0014$ & $.0002 \pm .0015$ & $-.0003 \pm .0015$ & $-.0001 \pm .0014$ \\
\hline
\end{tabular}

\begin{tabular}{|c|c|c|c|c|c|c|}
\hline & $r$ & $N$ & $\Sigma n$ & $\Sigma n / N$ & $r_{0}$ & $p_{0}$ \\
\hline Cape & $\begin{array}{l}\mathrm{s} \\
.0125\end{array}$ & 96 & 1489 & 15.5 & $\begin{array}{l}\mathrm{s} \\
.049\end{array}$ & 20 \\
\hline Heidelberg & .0126 & 97 & 1671 & 17.2 & .052 & 18 \\
\hline Washington & .0103 & 97 & 4318 & 45.5 & .070 & 10 \\
\hline Lick & .0237 & 97 & 507 & 5.2 & .054 & 17 \\
\hline Königsberg & .0123 & 83 & 1160 & 12.5 & .043 & 27 \\
\hline Edingburgh & .0128 & 94 & 1142 & 12,1 & .045 & 24 \\
\hline
\end{tabular}

Talule II.

Relative accuracy of former observation referred to the FK3 system.

$$
\text { where } r_{0}=\sqrt{\frac{\Xi n}{N}} r, \quad p_{0} \infty \frac{1}{r_{0}^{2}} \text {, }
$$


r........Probable error of the R.A. of each standard star referred to the KF3, system,

$N$.....Total number of the standard stars,

$\Sigma n$......Total number of observation for each standard star,

$\Sigma n / N \ldots$... Mean value of the number of observations for each standard star,

$r_{0} \quad$......Probable error of a single observation for each standard star,

$p_{0} \ldots$...Relative weight of a single observation for each standard star.

may be concluded that our relative determination of the right ascension has been carried out almost in accordance with the $F K 3$ system.

3. The determination of proper motion.

During a few years' period in the beginning of the present century, the observations of the zodiacal stars were carried out by the cooperation of the observatories in Europe and in America, and the results of the observations have been published in various catalog-forms. But the systems of the star places are not uniform, and the proper motions of the observed stars have not been determined. The clock stars we have adopted have been observed also in these catalogs. Hence, if we adopt these clock stars as the standard and calculate their places on the basis of the $F K 3$ system, we can reduce the results of the former observations to the $F K 3$ system and examine their systematic differences. Thus, by harmonic analysis, the following systematic differences are obtained. From the residua of the observed place of each star, the relative accuracy of the former observations is obtained as shown in Table I and Table II.

By applying the above systematic difference to the 97 clock stars of the former observations, we obtain a star catalog which contains 97 zodiacal fundamental stars observed in the beginning of the present century and reduced to the FK3 system. We call this catalog as ZOD 1900 provisionally, and the systematic difference between this catalog and the $F K 3$ has been again examined and tabulated in the last column of Table I. It is evident that, as ZOD 1900 is almost in accordance with the $F K 3$ system, we can also reduce the zodiacal stars other than our clock stars to the $F K 3$ system, by applying the systematic difference in Table I, if we neglect the effect of the magnitude equation. Thus we obtain the normal positions of the 2790 zodiacal stars reduced to the $F K 3$ system, the equinox being for 1900.0 with the observed epoch near 1900. Hence we get the proper motions of these stars by comparing these normal positions and the observed places obtained in our observatory.

4. The compilation of th- zodiacal catalog.

The observed places of the zodiacal stars have been reduced to the epoch 
1950.0 by applying the proper motions determined by the procedure just mentioned. Thus, a zodiacal star catalog has been compiled on the basis of the recent observations in our observatory, which we call the Tôkyô-Mitaka Zodiacal Star Catalog (TMZ 1950). It contains for each of the 2790 zodiacal stars, the right ascension at the epoch and the equinox 1950.0, with the first and the second terms of the centenial variation, the proper motion, the observed epoch, and the number of the observation, the approximate decliation at the epoch and the equinox 1950.0 with the first term of the centenial variation, its starname and identification of each star with the catalog number in the six important star catalogs, its Bessellian star number of each star at the epoch 1950.0, catalog difference of right ascension and proper motion of our catalog to $F K 3, G C, Z C$ (Washington, 1950).

5. Discussion of the catalog difference of proper motions.

By comparing the stability of the Repsold transit instrument and the cuccuracy of our observation, as described in the introduction of $T M Z$ catalog, with those of the modern observations made with the meridian circle, such as the observation of the zodiacal stars made with the 6 inch meridian circle of the Washington observatory 1928-1930, we see that the result of our observations is sufficiently accurate in spite of the size of the instrument employed. Hence we are fairly justified to conclude that the catalog difference between the two catalogs $T M Z$ and $Z C$ is not accidental. Now the catalog difference of proper motions described in the $T M Z$ catalog is expressed in the form

$$
\begin{aligned}
\frac{\Delta \alpha}{\Lambda t}(T M Z-Z C)=+ & \stackrel{\mathrm{s}}{0.0001 \sin \alpha+} \begin{array}{c}
\mathrm{s} \\
0.0002 \cos \alpha
\end{array} \\
& +0.0022 \sin 2 \alpha+0.0007 \cos 2 \alpha
\end{aligned}
$$

in which the predominating term is the one with $\sin 2 \alpha$ as the facter. The above results of observation and computation are shown in Fig. 1.

The calculated epochs of the proper motions of $T M Z$ and of $Z C$ are 1920 and 1900, respectively. Hence we can*look upon $\frac{\Lambda \alpha}{\Lambda t}(T M Z \sim Z C)$ as due to the variation of the proper motion during 20 years. Along the ecliptic we have $\tan \alpha=\cos \epsilon \tan \lambda$

$$
\frac{\Lambda \alpha}{\Lambda t}=-\frac{1}{2} \tan \epsilon \frac{\Lambda \epsilon}{\Lambda t} \sin 2 \alpha
$$

If we adopt $\frac{\Lambda_{\epsilon}}{\Lambda t}[20$ years] as the variation of $\epsilon$ in 20 years and neglect small terms on the right hand side of (1), we get

$$
-\frac{1}{2} \tan \epsilon \frac{\Lambda \epsilon}{\Lambda t}[20 \text { years }] \sin 2 \alpha=+0.0022 \sin 2 \alpha
$$


Hence the variation per year of $\epsilon$ is

$$
\frac{d \epsilon}{d t}=-0.0045
$$

When we correct the second term of Newcomb's constant of the obliquity of the ecliptic by an amount - 0.0045 , the catalog difference of the proper motions between $T M Z$ and $Z C$ is expected to disappear. Hense, as far as our observations are concerned, the second term of the obliquity $\epsilon$ of the ecliptic should be corrected to be -0.4729 while Newcomb's value for $\epsilon$ is -0.4684 . As the determination of $\epsilon$ by the solar observation is apt to be inaccurate, Newcomb himself and others have adopted the relative observations of asteroids and fixed stars, and Boss has referred only to the observations of fixed stars in the compilation of his fundarnental catalog. Our observations being relative to fundamental system, we can not determine the value of $\epsilon$ itself. However its variation is thus possibly determined when observation of equal accuracy made on different epoch are taken as the reference. Moreover, if we select fixed star near the ecliptic as the object of our observations, then we can regard the result as sufficiently accurate.

$$
\begin{aligned}
& \text { Real line ..... Obsemed value } \\
& \frac{\Delta \alpha}{\Delta t}(T M Z-Z G) \\
& \text { Broken line.... Computed value }
\end{aligned}
$$

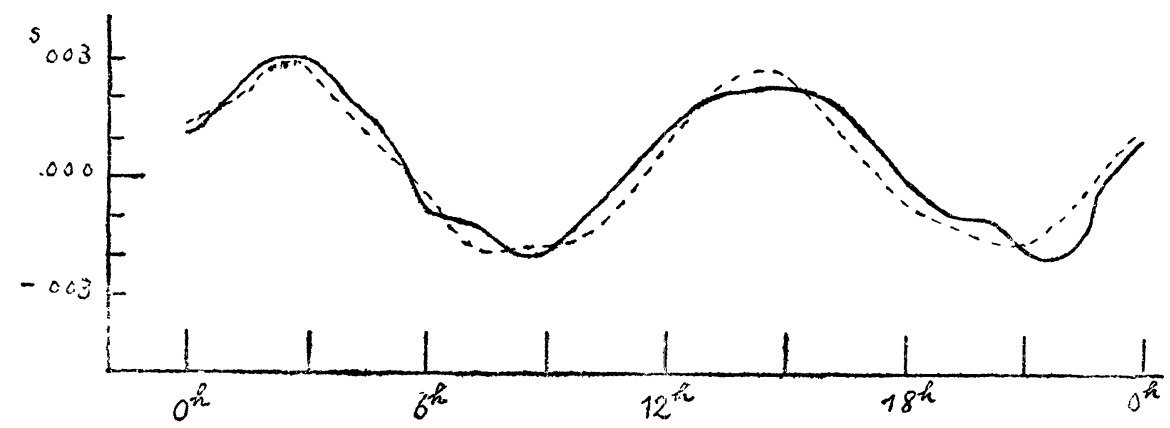

